\title{
Simulation of the DAMPE detector
}

\section{Wei Jiang, ${ }^{a, *}$ Zhan-Fang Chen, ${ }^{a, b}$ David Droz, ${ }^{c}$ Yi-Feng Wei ${ }^{d}$ and Yong-Jie Zhang ${ }^{e}$ on behalf of the DAMPE ${ }^{\dagger}$ Collaboration}

(a complete list of authors can be found at the end of the proceedings)

${ }^{a}$ Key Laboratory of Dark Matter and Space Astronomy, Purple Mountain Observatory, Chinese Academy of Sciences, Nanjing 210023, China

${ }^{b}$ School of Astronomy and Space Science, University of Science and Technology of China, Hefei 230026, China

${ }^{c}$ Università di Sassari, Dipartimento di Chimica e Farmacia, I-07100 Sassari, Italy

${ }^{d}$ Department of Modern Physics, University of Science and Technology of China, Hefei 230026, China

${ }^{e}$ Institute of Modern Physics, Chinese Academy of Sciences, Nanchang Road 509, Lanzhou 730000, China E-mail: jiangwei@pmo.ac.cn

Extensive Monte Carlo (MC) simulations are essential in understanding the detector's response for high energy particle detection experiments. We present the infrastructure and status of MC simulations of the DArk Matter Particle Explorer (DAMPE), a satellite project for the direct detection of high-energy cosmic rays and gamma rays. The DAMPE simulation tool employs two widely used softwares, GEANT4 and FLUKA, which implement various physics lists to simulate the interactions of particles in the detector. The framework of the simulation tool, the production farms, the data-MC comparison, and the performance of MC simulations on the analysis are summarized.

$37^{\text {th }}$ International Cosmic Ray Conference (ICRC 2021)

July 12 th - 23rd, 2021

Online - Berlin, Germany

\footnotetext{
${ }^{*}$ Presenter

${ }^{\dagger}$ The DAMPE mission is funded by the strategic priority science and technology projects in space science of Chinese Academy of Sciences. In China the data analysis is supported by the National Key Research and Development Program of China (No. 2016YFA0400200), the National Natural Science Foundation of China (Nos. 11525313, 11622327, 11722328, 11851305, 11921003, 12003076, U1738205, U1738206, U1738207, U1738208, U1738127, U1831206), the strategic priority science and technology projects of Chinese Academy of Sciences (No. XDA15051100), the Young Elite Scientists Sponsorship Program by CAST (No. YESS20160196), and the Program for Innovative Talents and Entrepreneur in Jiangsu. In Europe the activities and data analysis are supported by the Swiss National Science Foundation (SNSF), Switzerland, the National Institute for Nuclear Physics (INFN), Italy, and the European Research Council (ERC) under the European Union's Horizon 2020 research and innovation programme (No. 851103).
} 


\section{DArk Matter Particle Explorer}

The DArk Matter Particle Explorer (DAMPE; [1]), also known as "Wu-Kong", is a satellite based cosmic ray and $\gamma$-ray detector launched on December 17, 2015. The DAMPE aims to indirectly detect the annihilation or decay products of dark matter by observing cosmic ray electrons and $\gamma$-rays in a wide range of high-resolution energies. As a high energy cosmic ray detector, the DAMPE can also explore the origin of cosmic rays, as well as the transient high-energy $\gamma$-ray sky [2].

The DAMPE payload is made up of four sub-detectors, from top to bottom including a Plastic Scintillator Detector (PSD; [3]), a Silicon Tungsten tracKer-converter (STK; [4]), a Bismuth Germanium Oxide imaging calorimeter (BGO; [5]), and a NeUtron Detector (NUD; [6]). These four sub-detectors cooperate to measure the charge, direction, energy, and identity of each incident particle with high precision(see in Ref. [1] ). The on-orbit calibration in the first year proves that that DAMPE is fully operational as expected and all sub-detectors keep stable performance [7].

Extensive Monte Carlo (MC) simulations of the DAMPE detector with incident high energy particles are essential in understanding the detector's performance, such as the energy and direction resolution, the evaluations of detecting efficiency, and the background contaminations. The simulation software integrated into the DAMPE offline software is being used for large-scale production of events for scientific analyses. Up to now, based a huge mass of MC data, the DAMPE collaboration has reported high-precision measurements of the cosmic rays electron plus positron spectrum, proton spectrum and helium spectrum in wide energy ranges [8-10].

\section{DAMPE offline software overview}

The DAMPE offline software is the platform for data production and analysis, based on the DAMPE offline software framework [11] derived from the GAUDI framework [12] which was originally developed for $\mathrm{LHCb}$ and written in $\mathrm{C}++$. For the specific situation of the DAMPE collaboration, the DAMPE framework is designed as a light-weight, flexible and efficient to support various applications through basic classes and basic functions. The framework uses Python ${ }^{1}$ as the object-oriented script and interpreter language to configure and load $\mathrm{C}++$ algorithms and objects. Meanwhile, the data stream and store relies as much as possible on the ROOT [13], an open-source framework for data processing which was born at CERN and designed for high energy physics.

DAMPE offline software releases are divided into major modules according to functions, mainly including Kernel, Event, Simulation, Service, Calibration and Reconstruction. The Kernel contains the concrete implementation code of previous framework, is the most basic part of the entire program. Event module defines all the classes for data interaction and storage as ROOT object. Simulation module includes the code that calls the simulation software interface and describes the whole satellite. Service module aims at providing common functionality needed by the algorithms such as interface of geometry, database or configuration. Calibration module includes algorithms and parameters to calibrate each detecting unit from electronic signal to physical information. Reconstruction module provides algorithms to convert raw data into physical significance (energies, tracks, particle types, etc.).

\footnotetext{
${ }^{1}$ http://www.python.org
} 
All jobs running under the framework have three main steps, implemented by Python script called "job option". First, it is the initialization, which uses the dynamic library to load the required services and algorithms. In general, algorithms include methods that are called once per event, while services can be accessed multiple times in a single event. Configuration and initialization are controlled in a common Python infrastructure script, which allows introspection and is particularly useful for debugging and helping users. In addition, by using scripting language to load and configure objects, there is no need to recompile $\mathrm{C}++$ library or scripts for each job. Each algorithm and service can be configured differently for the specific step of data processing, allowing maximum sharing of common infrastructure among different steps. An algorithm can even be added many times as different sequence to the list of methods during an event loop.

Second, it is the loop the events to run algorithms. All the algorithms in the list run sequentially on each event. For example, you can add an event selection algorithm that filter some constraint events to this list. Other services and algorithms can also be called in the method. A messaging service invoked throughout the job controls the standard output with different levels of detail. Users can configure the total log detail level for a single algorithm or configure the detail level separately, which is particularly useful for debugging. An I/O service controls the data stream throughout the job from input to output, so that users do not need to take the I/O interaction into account, just focus on the algorithms instead.

Finally, at the end of the job, it is finalization step, that all algorithms are terminated and all objects are deleted. At this stage, the algorithms may output any statistical information they track (such as the filtering details of a event selection algorithm). Each job for data production is comprised by these three steps, and utilizes this infrastructure to provide users with maximum flexibility. Only the requested data are imported as branches and essential modules are loaded as plugins, so that each step consumes as little memory as possible and keeps as fast as possible during event loop.

\section{Detector simulation}

\subsection{Detector description}

As mentioned in Sect. 1, the DAMPE detector is described in detail in Ref. [1]. The MC simulation begins with the configuration of detector geometry. As shown in Fig. 1, a geometric model of the entire DAMPE payload accurately describes the detection units, the supporting structure and the filling cushioning materials of the sub-detectors, the frame and electronic components of the satellite.

The sizes, shapes, positions of all components adopt the designed parameters that are repeatedly measured and validated in detail during development and assembly of the satellite engineering. As for the materials, atomic composition of different elements are mainly been taken into account for high energy experiment of hadronic and electromagnetic shower cascade, while their chemical properties are ignored. The manufacturers provide detailed chemical components for most parts. The samples of the remaining materials which are classified as trade secrets are also be measured in detail for the exact mass fraction of atoms in analytical laboratories. 


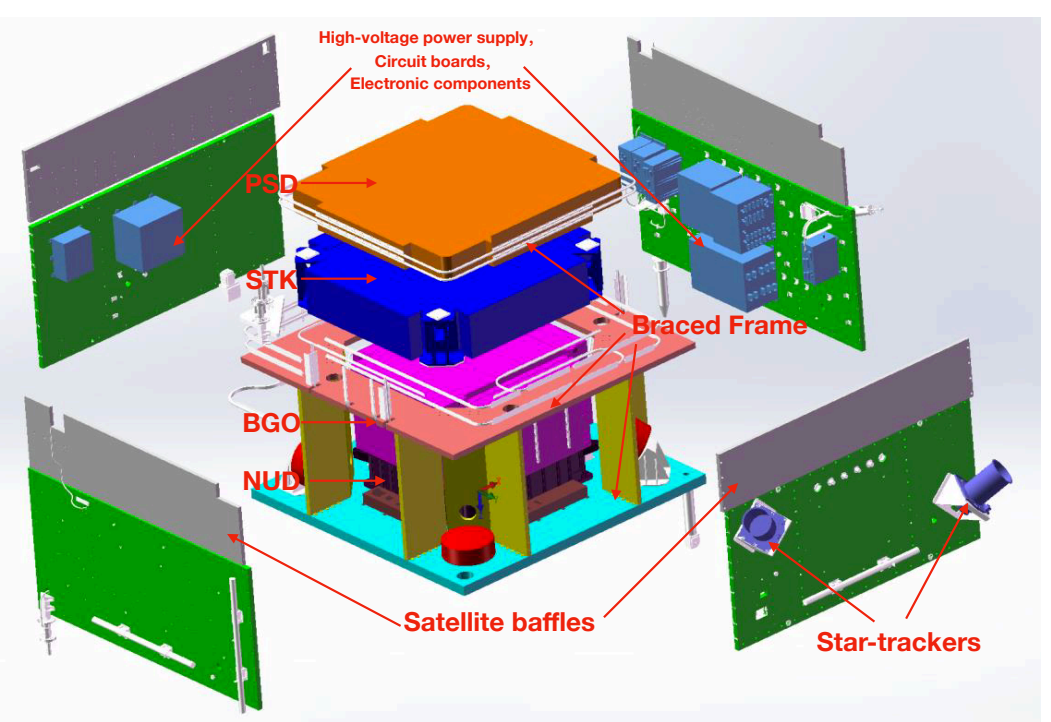

Figure 1: The visualization of the geometry of the DAMPE detector, including the payload and the entire satellite platform [14].

\subsection{GEANT4 simulation}

The DAMPE detector simulation employs the widely used Geant4 toolkit [15], a C++ framework, that includes a variety of physics models and data options for handling the interactions of particles with matter over a wide range of energy from $\mathrm{eV}$ scale to $\mathrm{TeV}$ scale. In that framework particles are generated according to a set of predetermined distributions (energy spectrum, spatial distribution) before they get propagated towards the detector material.

During each step interactions of the incident particle with its surrounding material are computed. In the case of DAMPE, the simulated geometry was derived from the CAD drawings of the instrument flight model and the generation of particles is chosen to mimic the on-orbit configuration, see Fig. 1, with particles impinging from all directions on the detector volume [16, 17]. In addition to Geant4, the Fortran-based simulation toolkits, including FLUKA [18] and CRMC [19], have been recently employed as well in the DAMPE software via custom Fortran-C++ interfaces. This allows the rigorous estimation of systematic uncertainties of cosmic ray measurements through a direct comparison of the results obtained with different hadronic models

The GEANT4 use a combination of ideas and approaches to perform all known particles and their interaction processes, callled "Physics List". There are several physics lists that are used in DAMPE simulation:

- QGSP_FTFP_BERT - officially recommended for astrophysical simulation, default option for electromagnetic processes.The list includes the Quark-Gluon String Precompound model (QGSP) [20] and the Bertini intranuclear cascade model (BERT) [21] as part of the hadronic physics package. 
- FTFP_BERT-the FRITOF Precompound model [22], default model for inelastic hadronnucleus processes over the energy range up to $100 \mathrm{TeV}$ in latest version. This $\mathrm{MC}$ results are mainly used for the measurement of cosmic ray proton and nuclei.

- FTFP_BERT_HP-identical to FTFP_BERT except that neutrons of $20 \mathrm{MeV}$ and lower use the High Precision neutron models and cross sections to describe elastic and inelastic scattering, capture and fission. Mainly used for the NUD detector for electron/proton identification up to $10 \mathrm{TeV}$.

The physics models used in the simulations are set as parameters in the DAMPE simulation framework, where the exact choice of the applied model depends on the considered energy range. While due to various limitations in the physics models used in Geant 4 we use the second model, namely EPOS-LHC [23] implemented by CRMC packsge [19] for generation events beyond 100 $\mathrm{TeV}$ energy range. The CRMC is connected in DAMPE simulation module through the GEANT4CRMC interface, as described in Ref. [24], developed with the great efforts of the researchers. We should stress that there is a significant difference between wall time usage at this energy range with respect to $10 \mathrm{TeV}-100 \mathrm{TeV}$. The reason is that the physics models used for the generation of $\mathrm{PeV}$ energy samples are fundamentally different from the lower energy ones, in particular, in the way the ion interactions are treated.

\subsection{FLUKA simulation}

FLUKA is a Fortran based, fully integrated and closed-source particle physics simulation package for calculations of particle transport and interactions with matter in the energy range from $\mathrm{MeV}$ up to PeV. It provides Fortran interface named Combinatorial Geometry (CG) to describe the geometry. After careful and repeated checks in comparison with the GDML geometry and the designed documents of satellite in every detail, we are confident that they are identical among each other and consistent with the real satellite geometry, although there are slight differences in some micro components of the satellite which are negligible during the simulation. The FLUKA code is also put in the simulation module as individual segment, and is performed to run proton and nuclei simulation up to $1 \mathrm{PeV}$ with the following settings:

- the PEANUT package is activated in the whole energy range for any reaction;

- the minimum kinetic energy for DPMJET-III is set to $5 \mathrm{GeV} / n$ (applying only to reactions between two nuclei heavier than a proton);

- the minimum kinetic energy for RQMD is set to $0.125 \mathrm{GeV} / n$ (applying only to reactions between two nuclei heavier than a proton)

\subsection{MC data production}

We have been producing simulation data for the entire duration of the satellite project. The simulation costs significant time consumption especially for energy above $100 \mathrm{TeV}$ that sometimes takes a few hours to finish an event. A huge mass of computing resources are required for the production. At present, the MC jobs are running at the collaboration's clusters in both Europe and China. In China, besides the computing clusters in collaboration with more than $2000 \mathrm{CPUs} / \mathrm{day}$, we 
also make use of some High Performance Computing (HPC) facilities out of DAMPE collaboration for our simulation tasks, including around 4000CPUs/day at the Sunway TaihuLight ${ }^{2}$ that was the fastest HPC in the world from 2016 to 2018 and flexible facilities up to ten thousand CPUs/day in a short tome at the Beijing Super Cloud Computing Center ${ }^{3}$ that is currently one of the top 3 Chinese HPCs. At present the above system is in place utilizing standard HPC computing farms at the Italian supercomputing farm CNAF (Tier $1{ }^{4}$ ) and Bari (Tier 3) as well as Baobab and Yggdrasil at University of Geneva, all of which are used in parallel for user activities (mainly data analysis, but other collaboration tasks as well). The Bari Tier 3 has also provided on average $300 \mathrm{CPUs} /$ day, whereas the CNAF pledge is about double that number (700 CPUs/day). Finally, Baobab and Yggdrasil at University of Geneva is a shared resource used by the entire department, providing on average $170 \mathrm{CPUs} /$ day for DAMPE activities. In addition to these farms we have support from the Swiss National Supercomputing Centre $\left(\mathrm{CSCS}^{5}\right)$ Cray XC50 - Multicore supercomputer. CSCS has proven to be an important resource for achieving groundbreaking results with DAMPE.

Overall, using over $7000 \mathrm{CPUs} /$ day and extensible HPCs, we have accomplished more than 40 billion MC events of the target particles from $\mathrm{GeV}$ to PeV. More MC samples above $100 \mathrm{TeV}$ are being produced and planned.

\section{MC data comparison}

An important reason why we spend so much doing so many different simulations is to determine the largest systemic uncertainties in cosmic ray nuclei energy spectrum measurements from the hadronic interaction models. The comparison of simulations of DAMPE between GEANT4 and FLUKA can be applied to determine the systemic uncertainties. For example, as shown in Fig. 2, the overall proton flux difference between GEANT4 and FLUKA is carried out. The proton flux difference varies from $-6.6 \%$ to $9.8 \%$, which is taken as the systematic uncertainty due to different hadronic interaction models.

\section{Summary}

We have presented the status and infrastructure of the DAMPE simulation, including framework, toolkit and farms. We have allocated massive computing resources to run, producing the simulation data of more than 40 billion of events for scientific analyses. Specially, different types of simulation software are implemented to determine the systematic uncertainties of the measurement of cosmic rays spectra.

\section{References}

[1] J. Chang et al. (DAMPE), Astroparticle Physics 95, 6 (2017).

[2] Q. Yuan and L. Feng, Sci. China Phys. Mech. Astron. 61, 101002 (2018), 1807.11638.

\footnotetext{
${ }^{2} \mathrm{http}: / /$ www.nsccwx.cn

${ }^{3}$ http://www.blsc.cn

${ }^{4}$ http://wlcg-public.web.cern.ch/tier-centres

${ }^{5}$ https://www.cscs.ch
} 


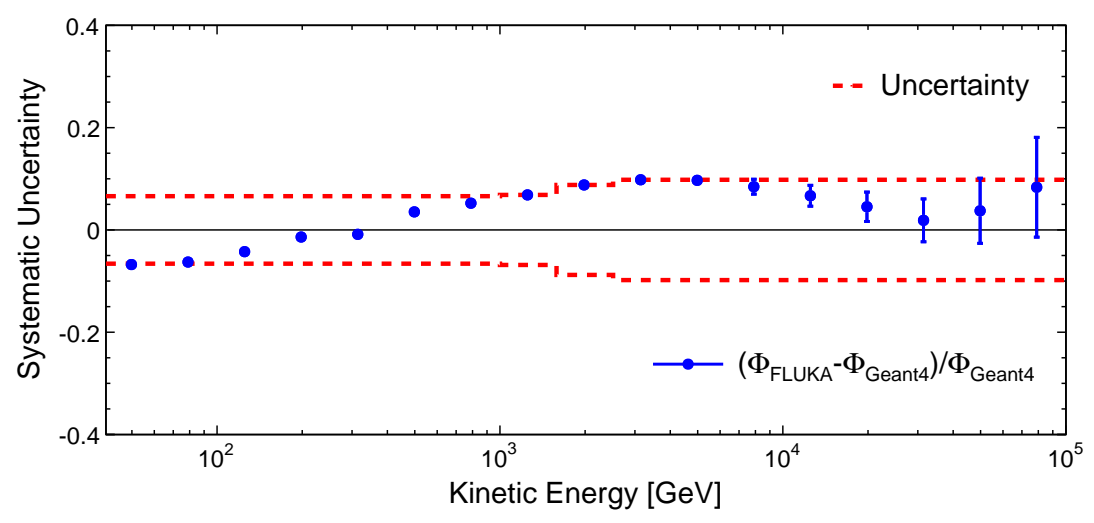

Figure 2: Energy dependence of the proton flux difference between GEANT4 and FLUKA [14] The blue points show the difference of measured proton spectrum assuming FLUKA simulation with respect to the spectrum based on GEANT4 simulation. The dashed lines correspond to the associated systematic uncertainty claimed in Ref. [9].

[3] Y. Yu et al., Astroparticle Physics 94, 1 (2017), 1703.00098.

[4] P. Azzarello et al., Nuclear Instruments and Methods in Physics Research A 831, 378 (2016).

[5] Z. Zhang et al., Nuclear Instruments and Methods in Physics Research A 836, 98 (2016).

[6] Y.-Y. Huang et al., Research in Astronomy and Astrophysics (RAA) 20, 153 (2020).

[7] G. Ambrosi et al. (DAMPE), Astroparticle Physics 106, 18 (2019), 1907.02173.

[8] G. Ambrosi et al. (DAMPE), Nature 552, 63 (2017).

[9] Q. An et al. (DAMPE), Science Advances 5, eaax3793 (2019), 1909.12860.

[10] F. Alemanno et al. (DAMPE), Phys. Rev. Lett. 126, 201102 (2021).

[11] C. Wang et al., Chinese Physics C 41, 106201 (2017).

[12] G. Barrand et al., Computer Physics Communications 140, 45 (2001).

[13] R. Brun and F. Rademakers, Nuclear Instruments and Methods in Physics Research A 389, 81 (1997), URL https://root.cern.ch.

[14] W. Jiang, C. Yue, M.-Y. Cui, et al., Chinese Physics Letters 37, 119601 (2020).

[15] S. Agostinelli, J. Allison, K. Amako, et al., Nuclear Instruments and Methods in Physics Research A 506, 250 (2003).

[16] A. Tykhonov, V. Gallo, X. Wu, and S. Zimme, J. Phys. Conf. Ser. 4, 042031 (2017).

[17] A. Tykhonov, A light-weight tool for converting cad drawings into the gdml format, https: //github.com/tihonav/cad-to-geant4-converter. 
[18] T. T. Böhlen, F. Cerutti, M. P. W. Chin, et al. (FLUKA Collaboration), Nuclear Data Sheets 120, 211 (2014).

[19] R. Ulrich, T. Pierog, and C. Baus, Cosmic Ray Monte Carlo Package (CRMC), https: // zenodo.org/record/4558706 (2021).

[20] A. Kaidalov and K. Ter-Martirosyan, Physics Letters B 117, 247 (1982).

[21] D. Wright and M. Kelsey, Nuclear Instruments and Methods in Physics Research Section A 804, 175 (2015).

[22] B. Nilsson-Almqvist and E. Stenlund, Computer Physics Communications 43, 387 (1987), ISSN 0010-4655.

[23] T. Pierog, I. Karpenko, J. Katzy, et al., Phys. Rev. C 92, 034906 (2015).

[24] A. Tykhonov et al., in Proceedings of 36th International Cosmic Ray Conference - PoS(ICRC2019) (2019), vol. 358, p. 143. 


\section{Full Authors List: DAMPE Collaboration}

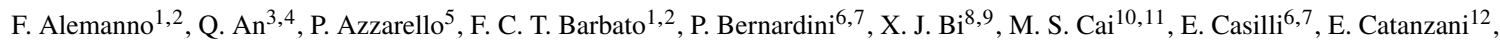
J. Chang ${ }^{10,11}$, D. Y. Chen ${ }^{10}$, J. L. Chen ${ }^{13}$, Z. F. Chen ${ }^{10,11}$, M. Y. Cui ${ }^{10}$, T. S. Cui ${ }^{14}$, Y. X. Cui ${ }^{10,11}$, H. T. Dai ${ }^{3,4}$, A. De Benedittis ${ }^{6,7}$, I. De Mitri ${ }^{1,2}$, F. de Palma ${ }^{6,7}$, M. Deliyergiyev ${ }^{5}$, M. Di Santo ${ }^{1,2}$, Q. Ding ${ }^{10,11}$, T. K. Dong ${ }^{10}$, Z. X. Dong ${ }^{14}$, G. Donvito ${ }^{15}$, D. Droz ${ }^{5}$, J.

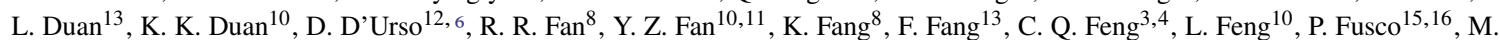

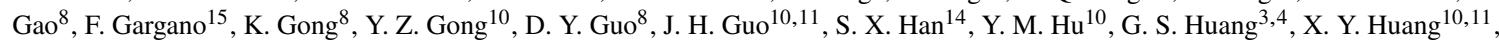

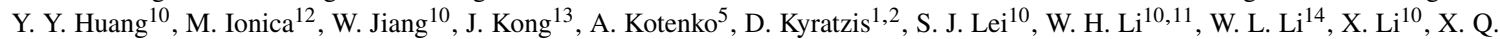

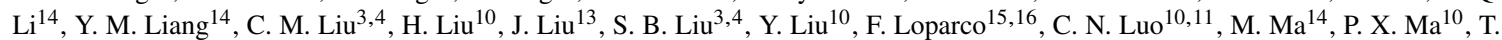

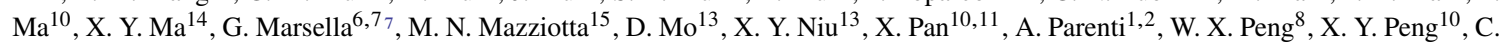
Perrina $^{5,8}$, R. Qiao ${ }^{8}$, J. N. Rao ${ }^{14}$, A. Ruina ${ }^{5}$, M. M. Salinas ${ }^{5}$, G. Z. Shang ${ }^{14}$, W. H. Shen ${ }^{14}$, Z. Q. Shen ${ }^{10}$, Z. T. Shen ${ }^{3,4}$, L. Silveri ${ }^{1,2}$, J. X. Song ${ }^{14}$, M. Stolpovskiy ${ }^{5}$, H. Su ${ }^{13}$, M. Su${ }^{17}$, H. R. Sun ${ }^{3,4}$, Z. Y. Sun ${ }^{13}$, A. Surdo ${ }^{7}$, X. J. Teng ${ }^{14}$, A. Tykhonov ${ }^{5}$, H. Wang ${ }^{14}$, J. Z.

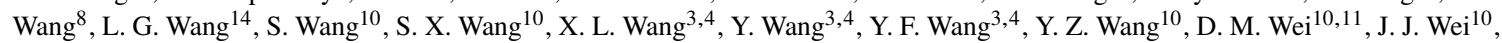
Y. F. Wei ${ }^{3,4}$, D. $\mathrm{Wu}^{8}$, J. Wu ${ }^{10,11}$, L. B. $\mathrm{Wu}^{1,2}$, S. S. $\mathrm{Wu}^{14}$, X. Wu ${ }^{5}$, Z. Q. Xia ${ }^{10}$, H. T. Xu ${ }^{14}$, Z. H. Xu ${ }^{10,11}$, Z. L. Xu ${ }^{10}$, E. H. Xu ${ }^{3,4}$, Z. Z. Xu ${ }^{3,4}$, G. F. Xue ${ }^{14}$, H. B. Yang ${ }^{13}$, P. Yang ${ }^{13}$, Y. Q. Yang ${ }^{13}$, H. J. Yao ${ }^{13}$, Y. H. Yu ${ }^{13}$, G. W. Yuan ${ }^{10,11}$, Q. Yuan ${ }^{10,11}$, C. Yue ${ }^{10}$, J. J. Zang $^{109}$ S. X. Zhang ${ }^{13}$, W. Z. Zhang ${ }^{14}$, Yan Zhang ${ }^{10}$, Yi Zhang ${ }^{10,11}$, Y. J. Zhang ${ }^{13}$, Y. L. Zhang ${ }^{3,4}$, Y. P. Zhang ${ }^{13}$, Y. Q. Zhang ${ }^{10}$, Z. Zhang ${ }^{10}$, Z. Y. Zhang ${ }^{3,4}$, C. Zhao ${ }^{3,4}$, H. Y. Zhao ${ }^{13}$, X. F. Zhao ${ }^{14}$, C. Y. Zhou ${ }^{14}$, and Y. Zhu ${ }^{14}$

${ }^{1}$ Gran Sasso Science Institute (GSSI), Via Iacobucci 2, I-67100 L'Aquila, Italy

${ }^{2}$ Istituto Nazionale di Fisica Nucleare (INFN) -Laboratori Nazionali del Gran Sasso, I-67100 Assergi, L’Aquila, Italy

${ }^{3}$ State Key Laboratory of Particle Detection and Electronics, University of Science and Technology of China, Hefei 230026, China

${ }^{4}$ Department of Modern Physics, University of Science and Technology of China, Hefei 230026, China

${ }^{5}$ Department of Nuclear and Particle Physics, University of Geneva, CH-1211, Switzerland

${ }^{6}$ Dipartimento di Matematica e Fisica E. De Giorgi, Universita“ del Salento, I-73100, Lecce, Italy

${ }^{7}$ Istituto Nazionale di Fisica Nucleare (INFN) - Sezione di Lecce, I-73100, Lecce, Italy

${ }^{8}$ Institute of High Energy Physics, Chinese Academy of Sciences, Yuquan Road 19B, Beijing 100049, China

${ }^{9}$ University of Chinese Academy of Sciences, Yuquan Road 19A, Beijing 100049, China

${ }^{10}$ Key Laboratory of Dark Matter and Space Astronomy, Purple Mountain Observatory, Chinese Academy of Sciences, Nanjing 210023, China

${ }^{11}$ School of Astronomy and Space Science, University of Science and Technology of China, Hefei 230026, China

${ }^{12}$ Istituto Nazionale di Fisica Nucleare (INFN) - Sezione di Perugia, I-06123 Perugia, Italy

${ }^{13}$ Institute of Modern Physics, Chinese Academy of Sciences, Nanchang Road 509, Lanzhou 730000, China

${ }^{14}$ National Space Science Center, Chinese Academy of Sciences, Nanertiao 1, Zhongguancun, Haidian district, Beijing 100190, China

${ }^{15}$ Istituto Nazionale di Fisica Nucleare (INFN) - Sezione di Bari, I-70125, Bari, Italy

${ }^{16}$ Dipartimento di Fisica "M. Merlin" dell'Universita' e del Politecnico di Bari, I-70126, Bari, Italy

${ }^{17}$ Department of Physics and Laboratory for Space Research, the University of Hong Kong, Pok Fu Lam, Hong Kong SAR, China

${ }^{6}$ Now at Dipartimento di Chimica e Farmacia, Universita' di Sassari, I-07100, Sassari, Italy.

${ }^{7}$ Now at Dipartimento di Fisica e Chimica "E. Segre", Universita“ degli Studi di Palermo, via delle Scienze ed. 17, I-90128 Palermo, Italy.

${ }^{8}$ Also at Institute of Physics, Ecole Polytechnique Federale de Lausanne (EPFL), CH-1015 Lausanne, Switzerland.

${ }^{9}$ Also at School of Physics and Electronic Engineering, Linyi University, Linyi 276000, China. 